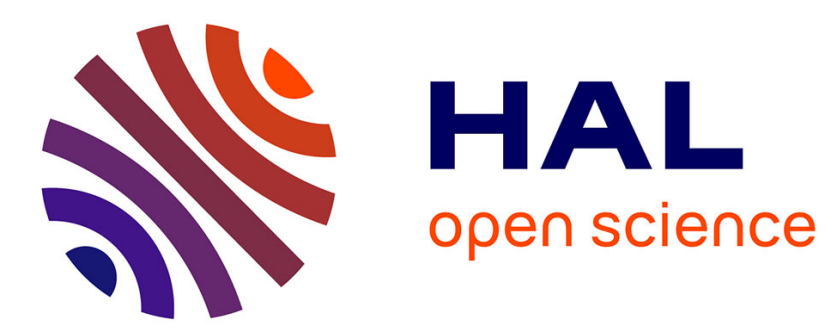

\title{
Competing structural orderings and transitions to glass in mixed crystals of $\mathrm{Rb} 1-\mathrm{x}(\mathrm{NH} 4) \mathrm{xH} 2 \mathrm{PO} 4$
}

\author{
E. Courtens
}

\section{To cite this version:}

E. Courtens. Competing structural orderings and transitions to glass in mixed crystals of Rb1-x(NH4)xH 2PO4. Journal de Physique Lettres, 1982, 43 (6), pp.199-204. 10.1051/jphyslet:01982004306019900 . jpa-00232031

\section{HAL Id: jpa-00232031 https://hal.science/jpa-00232031}

Submitted on 1 Jan 1982

HAL is a multi-disciplinary open access archive for the deposit and dissemination of scientific research documents, whether they are published or not. The documents may come from teaching and research institutions in France or abroad, or from public or private research centers.
L'archive ouverte pluridisciplinaire HAL, est destinée au dépôt et à la diffusion de documents scientifiques de niveau recherche, publiés ou non, émanant des établissements d'enseignement et de recherche français ou étrangers, des laboratoires publics ou privés. 
Classification

Physics Abstracts

$61.40 \mathrm{D}-77.80 \mathrm{D}-64.70 \mathrm{E}-78.20 \mathrm{~F}$

\title{
Competing structural orderings and transitions to glass in mixed crystals of $\mathrm{Rb}_{1-x}\left(\mathrm{NH}_{4}\right)_{x} \mathrm{H}_{2} \mathrm{PO}_{4}$
}

\author{
E. Courtens \\ IBM Zurich Research Laboratory, 8803 Rüschlikon-ZH, Switzerland
}

(Reçu le 17 décembre 1981, accepté le 25 janvier 1982)

\begin{abstract}
Résumé. - On produit de la frustration dans des cristaux de la famille du KDP par mélange de composants aux tendances ferroélectriques et antiferroélectriques. Des mesures diélectriques et de biréfringence sont présentées pour $0 \leqslant x \leqslant 0,35$. Elles indiquent un diagramme de phase intéressant, avec une phase vitreuse précédée probablement par une phase mixte pour les concentrations élevées. La compétition au hasard d'interactions à courte portée joue sans doute un rôle important dans ce système, rendant ces verres de véritables analogues structuraux des verres de spin magnétiques.
\end{abstract}

\begin{abstract}
Frustration is produced in crystals of the KDP family by mixing ferroelectric and antiferroelectric constituents. Birefringence and dielectric measurements are reported for $0 \leqslant x \leqslant 0.35$. They indicate an interesting phase diagram, with a glassy phase probably preceded by a mixed phase for the upper part of the concentration range investigated. The randomness of the competing shortrange interactions is likely to play a major role, making these structural glasses truly analogous to magnetic spin glasses.
\end{abstract}

Considerable attention is currently being given to systems with random frustration [1]. Experimentally, the interest has concentrated on magnetic spin glasses [2]. Dipolar glasses [3], with frustrated random dipolar interactions [4], have also been investigated. As it would be of interest to know a structural analog of exchange-coupled spin glasses [5], we take here a fresh look at crystals with mixed short-range ferroelectric (FE) and antiferroelectric (AFE) constituents [6]. An experimental study of $\mathrm{Rb}_{1-x}\left(\mathrm{NH}_{4}\right)_{x} \mathrm{H}_{2} \mathrm{PO}_{4}$ is presented for $0 \leqslant x \lesssim 0.35$. Detailed information is given on the upper concentration range, where evidence for a glass transition probably preceded by a mixed phase is obtained.

$\mathrm{RbH}_{2} \mathrm{PO}_{4}$ (RDP) and $\mathrm{NH}_{4} \mathrm{H}_{2} \mathrm{PO}_{4}$ (ADP) form isomorphous tetragonal crystals at ambient temperature. Their lattice parameters match closely, and mixed crystals can apparently be grown over the full range of $x$ [7]. The $\mathrm{H}$-bonds linking $\mathrm{PO}_{4}$ tetrahedra play an important role in the transitions : the ordering of the acid protons in " up-down " Slater configurations [8] produces the FE transition in RDP, whereas " lateral » ordering generates the AFE phase of ADP. The latter presumably occurs because the $\mathrm{NH}_{4}^{+}$protons tend to form their own $\mathrm{H}$-bonds with four nearby $\mathrm{PO}_{4}$ groups [9]. This additional bonding manifests itself in many ways, in particular by a distribution coefficient which favours $\mathrm{NH}_{4}^{+}$incorporation in the growing crystal [7, 10]. The FE transition of RDP (at $T_{\mathrm{c}}=146 \mathrm{~K}$ ) is believed to be continuous [11]. The FE structure results from condensation of a mode of $B_{2}$ symmetry at the centre of the Brillouin zone [12] and the 
orthorhombic axes are rotated $45^{\circ}$ from the $a$-axes of the body-centred tetragonal cell. In ADP, the AFE transition $\left(T_{\mathrm{c}}=147 \mathrm{~K}\right)$ is strongly first-order. The orthorhombic distortion is that of an $\mathbf{M}_{34}$ mode at the $\mathrm{M}$-point [12], with axes parallel to the original tetragonal ones.

The investigation reported here was performed with birefringence, electro-optic, and dielectric measurements. The FE transition produces a spontaneous birefringence [13], given in first-order by $\Delta n_{12}=n_{0}^{3} \rho_{63} P_{3}$ [14]. The polarization along the FE axis is $P_{3}, n_{0}$ is an average refractive index, and $\rho_{63}$ is an electro-optic coefficient. As the latter is nearly constant, the measurement of $\Delta n_{12}$ gives access to the FE order parameter $P_{3}$. Thin plates were provided with semi-transparent electrodes allowing application of a poling field $E_{3}$. A low power $(\sim 20 \mu \mathrm{W})$, slightly focussed He-Ne laser beam (6328 $\AA$ ) was used. For small $x$, the paraelectric (PE) to FE transition temperature decreases rapidly with increasing $x, \mathrm{~d} T_{\mathrm{c}} / \mathrm{d} x \simeq-300 \mathrm{~K}$. The saturation polarization remains sizeable, however, practically equal to that of RDP when account is taken of the also observed small decrease of $\rho_{63}$ with increasing $x$. For $x \gtrsim 0.13, T_{\mathrm{c}}$ decreases even faster and the transition exhibits a first-order birefringence jump on cooling, whereas fringes can still be counted on heating back to the high-temperature phase. With $E_{3}=0$, the onset of the new phase is manifested by the sudden appearance of near-forward scattering in the form of a cross produced by domain walls [15]. Near $x=0.2$, it becomes impossible to pole the crystals with the highest fields applied $(\sim 300 \mathrm{kV} / \mathrm{m})$. For $x \simeq 0.22$, no optical evidence remains of any transition towards a phase of lower point symmetry. The field cooled value of $\Delta n_{12}$ can then be measured with a compensator. At low $E_{3}$, it is proportional to $E_{3}$, with $\Delta n_{12}=n_{0}^{3} r_{63} E_{3}$, and $r_{63} \equiv \rho_{63} \varepsilon_{0}\left(\varepsilon_{33}^{\prime}-1\right)$. Here, $\varepsilon_{0}$ is the dielectric permittivity of vacuum, and $\varepsilon_{33}^{\prime}$ is the real part of the dielectric constant along the FE axis. This is shown in figure 1 for $x \simeq 0.34$. Up to the highest field, $\Delta n_{12}$ remains considerably smaller than the values attained in the FE phase with $x<0.2$. The low-temperature

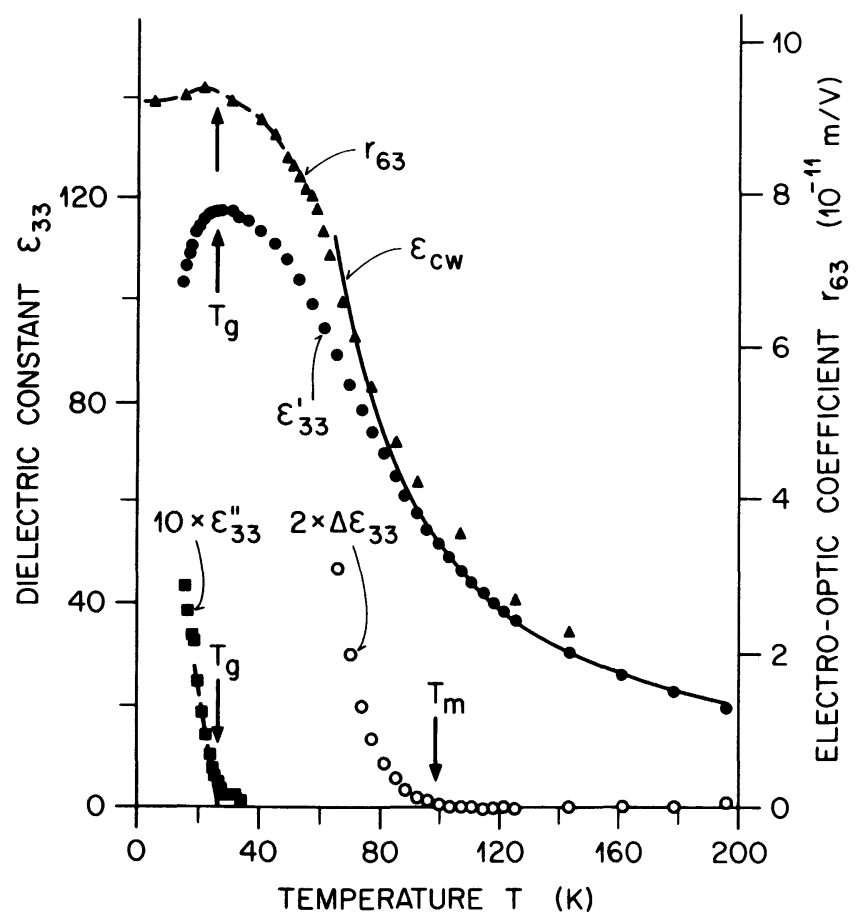

Fig. 1. - Right ordinate : the field cooled electro-optic coefficient $r_{63}:$ the dashed line is a guide to the eye. Left ordinate : the real and imaginary parts of the longitudinal dielectric constant at $1 \mathrm{kHz}, \varepsilon_{33}^{\prime}$ and $\varepsilon_{33}^{\prime \prime}$, respectively $(x=0.34)$. The solid line is the high-temperature Curie-Weiss fit, while $\Delta \varepsilon_{33}=\varepsilon_{\mathrm{CW}}-\varepsilon_{33}^{\prime}$. 
plateau of $r_{63}$, as well as thermal release of polarization and a number of effects indicating remanence, were suggestive of a transition to a glass phase with a random FE-type condensation.

To characterize the situation further, careful measurements of $\varepsilon_{33}$, the susceptibility associated with the FE phase of KDP, and of $\varepsilon_{11}$ were performed. Results at $1 \mathrm{kHz}$ are shown in figures 1 and 2. The temperature $T_{\mathrm{g}}$ marks the onset of dispersion and dielectric loss $\left(\varepsilon^{\prime \prime} \neq 0\right)$ in both $\varepsilon_{i i}(\omega)$. Below $T_{\mathrm{g}}, \varepsilon_{i i}^{\prime}(\omega)$ decreases markedly with increasing $\omega$ and decreasing $T$. Remembering that $r_{63}$ is proportional to $\varepsilon_{33}^{\prime}(\omega=0)$, the behaviour is strikingly similar to that of spin glasses [2]. All results are compatible with a random FE-type condensation below $T_{\mathrm{g}}$. For reasons explained below, we propose to name this new phase a « structural glass » $(\mathrm{SG})$.

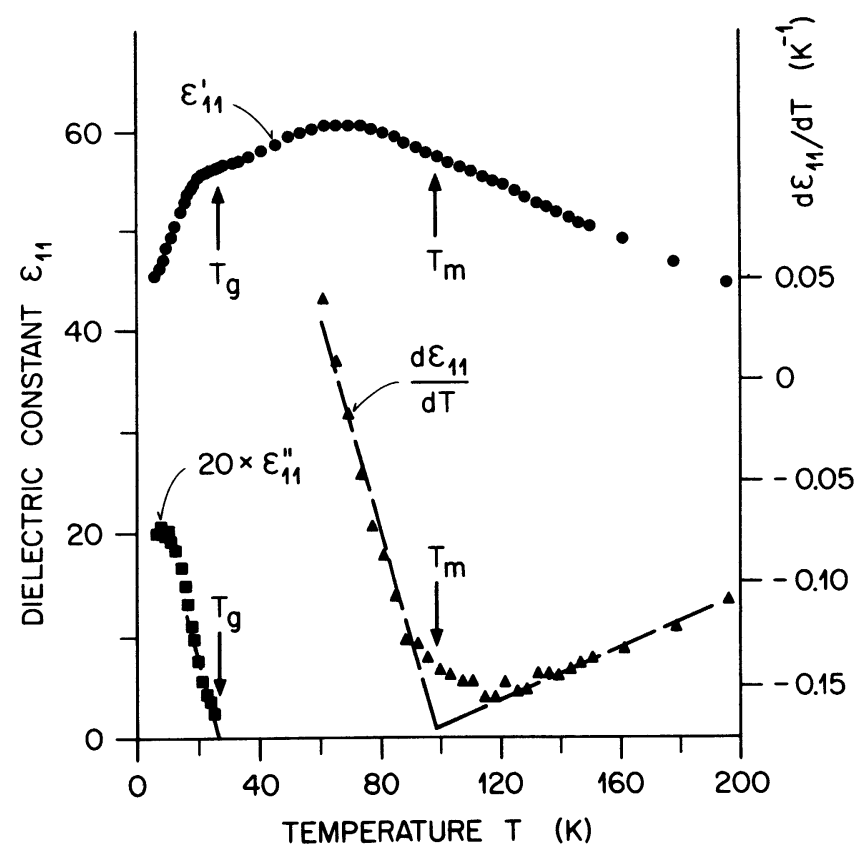

Fig. 2. - Left ordinate : the real and imaginary parts of the transverse dielectric constant at $1 \mathrm{kHz}, \varepsilon_{11}^{\prime}$ and $\varepsilon_{11}^{\prime \prime}$, respectively (for $x=0.34$ ). Right ordinate : the derivative of $\varepsilon_{11}^{\prime}$ obtained with a three-point algorithm.

Quite salient is the broad maximum in $\varepsilon_{11}$ which occurs much above $T_{\mathrm{g}}$. The transverse susceptibility derives a large contribution from the polarizability of the acid-proton bonds, which are mainly perpendicular to $c$. This maximum indicates they have reached a degree of ordering which counteracts their ability to contribute to $P_{1}$ by changing their distribution among the potential wells. A clue to the type of ordering they adopt is given by $\varepsilon_{33}$. In the PE phase of RDP, $\varepsilon_{33}$ follows closely a mean-field Curie-Weiss law [11], and the same is found here at sufficiently high temperatures, above $T_{\mathrm{m}}=99 \mathrm{~K}$. The Curie-Weiss temperature obtained by fitting $\varepsilon_{33}$ from 300 to $100 \mathrm{~K}$ is $T_{\mathrm{Cw}}=38 \mathrm{~K}$, significantly higher than $T_{\mathrm{g}}=27 \mathrm{~K}$. Below $T_{\mathrm{m}}, \varepsilon_{33}$ is smaller than the extrapolated Curie-Weiss value $\varepsilon_{\mathrm{CW}}$; both $\varepsilon_{\mathrm{CW}}$ and the difference $\Delta \varepsilon_{33}=\varepsilon_{\mathrm{CW}}-\varepsilon_{33}$ are shown in figure 1 . This indicates that progressively more sites are prevented from contributing to ferroelectricity, which also forces $T_{\mathrm{g}}$ below $T_{\mathrm{Cw}}$. As the effect is not accompanied by appreciable dielectric loss or dispersion, it is reasonable to infer a random AFE-type condensation. Indeed, antiferroelectric clusters do not couple linearly to the applied electric field. The formation of such clusters can be thought as preventing a fraction of the material from contributing to the 
growth of $\varepsilon_{33}$ and $\varepsilon_{11}$ on cooling. Remarkably, $T_{\mathrm{m}}$ also corresponds to the inflection point of $\varepsilon_{11}$, which is better seen in the numerical derivative plotted in figure 2.

To further support this conjecture, the change in the natural birefringence of the tetragonal phase [13], $\Delta\left(n_{\mathrm{o}}-n_{\mathrm{e}}\right)$, was also measured. Here, $n_{\mathrm{o}}$ and $n_{\mathrm{e}}$ are the ordinary and extraordinary indices, respectively. This change has two causes. Firstly, the lattice anharmonicity produces thermal expansion, leading to a variation of both indices, and of their difference, proportional to density changes. For temperatures above the proton ordering, excellent fits to a Debye function are obtained, with a satisfactory Debye temperature of $\sim 400 \mathrm{~K}$. Extrapolating the Debye function to lower temperatures, and subtracting the observed $\Delta\left(n_{\mathrm{o}}-n_{\mathrm{e}}\right)$, one obtains a remainder $\delta\left(n_{\mathrm{e}}-n_{\mathrm{o}}\right)$. In pure KDP, this remainder is known to be proportional to $P_{3}^{2}$, and we find this result also for RDP [13]. In pure ADP, for similar symmetry reasons, $\delta\left(n_{\mathrm{e}}-n_{\mathrm{o}}\right)$ should be proportional to the square of the AFE order parameter. The onset of $\delta\left(n_{\mathrm{e}}-n_{\mathrm{o}}\right)$ on cooling, in the absence of any other symmetry change of the crystal, can thus be interpreted as the development of an Edwards-Anderson order parameter $q$ [16]. Approximately, one would have $q \propto \delta\left(n_{\mathrm{e}}-n_{\mathrm{o}}\right)$. As shown in figure 3 , there is indeed an onset at $T_{\mathrm{m}}$. Interestingly, no noticeable change occurs at $T_{\mathrm{g}}$. The solid curve is an empirical fit to $q=3 \tau^{2}-2 \tau^{3}$, where $\tau \equiv 1-T / T_{\mathrm{m}}$. This appears significantly different from the dependence $q=\tau+\tau^{2}-\tau^{3}$ predicted for the SherringtonKirkpatrick (SK) Hamiltonian [17]. It suggests that another model might be needed to account for the particular order parameter and/or randomness of the present case. Careful examination of our data at other concentrations indicates the occurrence of this condensation at least for all $x \gtrsim 0.13$. Tentatively, the new phase, if there is one, will be called a mixed phase (M), as roughlyspeaking it corresponds to a mixture of paraelectric regions with AFE clusters.

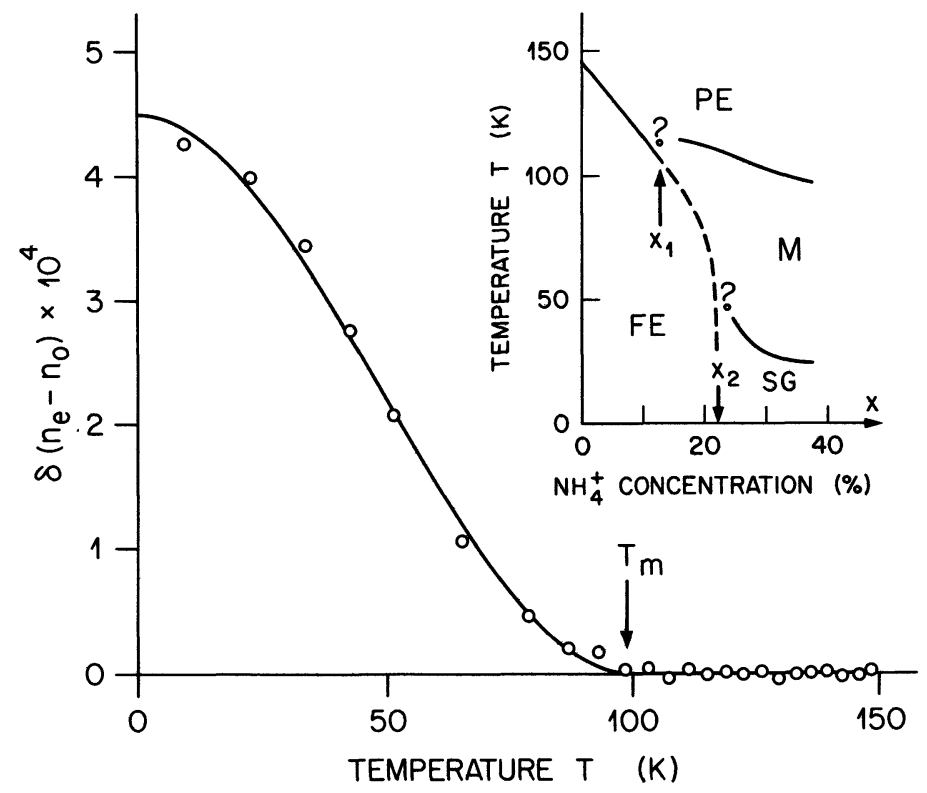

Fig. 3. - The remainder of the tetragonal birefringence $\delta\left(n_{\mathrm{e}}-n_{\mathrm{o}}\right)$ after subtraction of the lattice expansion contribution for $x=0.34$. The solid line is the fit explained in the text. Inset : A tentative phase diagram. Along the dashed line, the transition is clearly first-order, while it seems continuous elsewhere.

A provisional phase diagram summarizing the present information is shown in the inset of figure 3. The nature of the special points remains to be elucidated. The occurrence of the $\mathbf{M}$ region before the SG phase is in line with the recent theoretical result on the Heisenberg SK model in presence of a field [18]. Here, no field needs to be applied to reveal the $\mathbf{M}$ region, as the aniso- 
tropy required is provided by the crystal itself. The first-orderness of the $M \rightarrow F E$ transition indicates a strong competition between the two phases, which is easy to understand intuitively. It should be noted that approximate values for the concentrations $x_{1}$ and $x_{2}$ of figure 3 can be derived by simple percolation reasoning. Considering the $\mathrm{PO}_{4}$ groups as single units, one finds that they are linked by the acid-proton bonds with the topology of the diamond lattice. $\mathrm{Each}_{\mathrm{NH}}^{+}$ perturbs four $\mathrm{PO}_{4}$ groups forming a tetrahedron of second-nearest neighbours, and conversely, each $\mathrm{PO}_{4}$ is surrounded by four sites available to $\mathrm{NH}_{4}^{+}$perturbers. For random substitution, the probability of a $\mathrm{PO}_{4}$ site to remain unperturbed is simply $(1-x)^{4}$. We surmise that the percolation of perturbed $\mathrm{PO}_{4}$ groups forces the protons to seek an arrangement leading to AFE-type condensation. Neglecting correlations, the site percolation threshold in the diamond lattice is 0.43 [19], giving $0.43 \simeq 1-\left(1-x_{1}\right)^{4}$, or $x_{1} \simeq 13 \%$, in agreement with observation. On the other hand, the FE phase could be suppressed when the unperturbed groups cease to percolate. Again, neglecting the correlations of perturbed sites, this gives $0.43 \simeq\left(1-x_{2}\right)^{4}$, or $x_{2} \simeq 19 \%$, in reasonable agreement with the findings. This emphasizes the possible importance of shortrange interactions, but does not prevent long-range models from applying. Indeed, the order parameter $q$ is, by symmetry, linearly coupled to density, and that coupling can have a strong fluctuation-quenching effect due to elasticity, as is now well known for KDP [20]. Since $\delta\left(n_{\mathrm{e}}-n_{\mathrm{o}}\right)$ is also expected to be linearly related to density changes, at least the $\mathrm{PE} \rightarrow \mathrm{M}$ transition might be describable adequately by some mean-field approach. A genuine structural analog of magnetic spin glasses seems to have been discovered here [21]. The system could be called either a ferroelectric, a polar, or a structural glass. The last-mentioned name is preferred. The first one commonly refers to non-crystalline ferroelectrics, and the second one has been used for situations where dipolar interactions were thought important, which is presumably not the case presently, as explained above.

H. J. Scheel contributed by his careful crystal growth and in discussions, P. Bayer patiently helped with delicate technical aspects, and $\mathrm{H}$. Weibel provided some exploratory $\varepsilon_{33}$ measurements and technical advice. Many thanks are expressed to all of them, as well as to A. Baratoff, K. A. Müller, D. Sherrington and R. H. Swendsen for stimulating conversations.

\section{References}

[1] For recent theoretical reviews, see Anderson, P. W., J. Appl. Phys. 49 (1978) 1599 ;

Brézin, E., Gervais, J. L. and Toulouse, G., Phys. Rep. 67 No. 1 (1980);

BINDER, K., Proceedings of the EPS Conference on Condensed Matter, Antwerp, 1980 (Plenum Press, New York) to be published.

[2] Mydosh, J. A., Proceedings of the International Conference on Disordered Systems and Localization, Rome, 1981 (Springer, Heidelberg) to be published, and references therein.

[3] Kanzig, W., Hart, H. R. and Roberts, S., Phys. Rev. Lett. 13 (1964) 543.

[4] Fischer, B. and Klein, M. W., Phys. Rev. Lett. 37 (1976) 756.

[5] Maletta, H. and Felsch, W., Phys. Rev. B 20 (1979) 1245.

[6] Matthias, B. and Merz, W., Helv. Phys. Acta 19 (1946) 227.

[7] SCheel, H. J. and Courtens, E., to be published.

[8] Slater, J. C., J. Chem. Phys. 9 (1941) 16.

[9] Megaw, H. D., Ferroelectricity in Crystals (Methuen, London) 1957, Chap. 3.

[10] For this reason, $x$ had to be determined carefully. As the density is a sensitive function of $x$, flotation was used and confirmed by X-ray powder analysis. The latter did not indicate superstructures.

[11] Bastie, P., Lajzerowicz, J. and Schneider, J. R., J. Phys. C 11 (1978) 1203 and references therein.

[12] BLINC, R. and ZeKs, B., Soft Modes in Ferroelectrics and Antiferroelectrics (North-Holland, Amsterdam) 1974, p. 94-96.

[13] Zwicker, B. and Scherrer, P., Helv. Phys. Acta 17 (1944) 346 ;

Vallade, M., Phys. Rev. B 12 (1975) 3755. 
[14] Abbreviated subscripts are used, and the axes are those of the tetragonal phase throughout.

[15] See, e.g. Courtens, E., J. Phys. C 14 (1981) L-37.

[16] Edwards, S. F. and ANDerson, P. W., J. Phys. F 5 (1975) 965.

[17] Parisi, G. and Toulouse, G., J. Physique Lett. 41 (1980) L-361.

[18] Gabay, M. and Toulouse, G., Phys. Rev. Lett. 47 (1981) 201.

[19] Stauffer, D., Phys. Rep. 54 (1979) 1.

[20] Courtens, E., Gammon, R. and Alexander, S., Phys. Rev. Lett. 43 (1979) 1026.

[21] Another example might be provided by the « rotational » system $\mathrm{KBr}_{1-x}(\mathrm{CN})_{x}$ [Rowe, J. M., RuSH, J. J., Hinks, D. G. and Susman, S., Phys. Rev. Lett. 43 (1979) 1158, and references therein], if it turns out to be a real glass. 